\title{
Wound healing effect of regenerated oxidized cellulose versus fibrin sealant patch: An in vivo study
}

\author{
Hyun Sik Yoon ${ }^{1}$, \\ Young Cheon $\mathrm{Na}^{1}$, \\ Keum Ha Choi², \\ Woo Hoe Huh ${ }^{1}$, \\ Ji Min Kim ${ }^{1}$
}

Departments of ${ }^{1}$ Plastic and Reconstructive Surgery and ${ }^{2}$ Pathology, Wonkwang University Hospital, Iksan, Korea
Background: Topical hemostatic agents are used when ligation, electrocauterization, or other conventional hemostatic methods are impractical. Because a hemostatic agent is a foreign body, it can cause foreign body reactions, inflammation, and infections that can interfere with the wound healing process. Therefore, we should select hemostatic agents after considering their effects on wound healing. Here, we compared the effects of hemostatic agents on wound healing in a rectus abdominis muscle defect in rats.

Methods: Twelve Sprague Dawley rats were subjected to creation of a $6 \times 6 \mathrm{~mm}$ defect in the rectus abdominis muscle and divided into four groups: control group; group A, Tachosil fibrin sealant patch; group B, Surgicel Fibrillar oxidized regenerated cellulose; and group C, Surgicel Snow oxidized regenerated cellulose. For the histologic analysis, biopsies were performed on the $3 r d$, 7th, and 27th days.

Results: The foreign body reaction was the weakest in group $A$ and most significant in group $C$. The inflammatory cell infiltration was the weakest in group $A$ and similar in groups $B$ and $C$. Muscle regeneration differed among periods. The rats in group $A$ were the most active initially, while those in group $\mathrm{C}$ showed prolonged activity.

Conclusion: Tachosil and Surgicel administration increased inflammation via foreign body reactions, but the overall wound healing process was not significantly affected. The increased inflammation in the Surgicel groups was due to a low pH. We recommend using Tachosil, because it results in less intense foreign body reactions than Surgicel and faster wound healing due to the fibrin action.

Keywords: Cellulose / Fibrin / Fibrinogen / Hemostatics / Thrombin / Wound healing

\section{INTRODUCTION}

Topical hemostatic agents are applied when compression, ligation, electrocauterization, or other conventional hemostatic

Correspondence: Young Cheon Na

Department of Plastic and Reconstructive Surgery, Wonkwang University Hospital, 895 Muwang-ro, Iksan 54538, Korea

E-mail: ycnadr@hanmail.net

This work was supported by Wonkwang University in 2019.

Received September 11, 2019 / Revised October 11, 2019 / Accepted October 17, 2019 methods are impractical [1,2]. Topical hemostatic agents are currently used to reduce blood loss and hemostasis time, avoid the side effects of systemic hemostatic drugs, and ultimately shorten surgery time to improve patient condition and reduce complications and cost $[2,3]$. When choosing a hemostatic agent, we usually focus on how fast and effective the hemostasis appears. Of course, it is desirable to have minimum inflammation without foreign body reaction and delay of the wound healing process after the application of topical hemostatic 
agents. However, because it is a foreign body, its use can cause foreign body reactions, inflammation, and infections, which can interfere with wound healing process [2]. Therefore, surgeons should carefully select hemostatic agents upon considering its mechanism, advantages, disadvantages, and effects on wound healing. The structure of rat skin is very similar to that of human skin. Rat skin is composed of epidermis, dermis, and subcutaneous striated muscles from the outside, and the epithelial appendages are mostly composed of hair follicles and sebaceous glands. Hair follicles play an important role in the epithelization process [4]. We compared the effect of hemostatic agents on wound healing by applying them to a rectus abdominis muscle defect in rats. This study aimed to provide information to guide surgeons' selection of topical hemostatic agents.

\section{METHODS}

The experimental protocol, including the use of animals in the research, was approved by the Institutional Animal Care and Use Committee of Wonkwang University.

\section{Materials}

\section{Experimental animals}

Twelve male 9-week-old Sprague Dawley rats weighting 280$300 \mathrm{~g}$ each were used in this study. After a 1-week acclimatization period, the rats were randomly divided into four groups of three animals each. Each rat was housed separately (one per cage) and maintained on a standard pellet diet and tap water.

\section{Experimental materials}

A total of three experimental materials were used: Tachosil absorbable fibrin sealant patch (Baxter, Deerfield, IL, USA); Surgicel Fibrillar absorbable oxidized regenerated cellulose (Johnson \& Johnson, New Brunswick, NJ, USA); and Surgicel Snow absorbable oxidized regenerated cellulose (Johnson \& Johnson).

\section{Methods}

\section{Wound induction}

After a 1-week acclimatization period, 12 male Sprague Dawley rats were anesthetized by isoflurane and sevoflurane (Baxter) for inhalation anesthesia. The fur was shaved with electrical shaver and disinfected with $10 \%$ povidine iodine and $70 \%$ alcohol. We made an incision with a no. 15 scalpel and scissors at the abdominal midline and then dissected the subcutaneous tissue and made four $6 \times 6 \mathrm{~mm}$ uniform muscle defects in both rectus abdominis muscles using a 6-mm disposable biopsy punch (Kai Medical, Chiyoda, Japan) and a no. 15 scalpel.

\section{Wound care}

Twelve male Sprague Dawley rats were randomly divided into four groups of three animals each: control group, no care was provided for the muscle defect; group A, the muscle defect was treated with a Tachosil absorbable fibrin sealant patch according to the manufacturer's instructions; group B, the muscle defect was treated with a Surgicel Fibrillar absorbable oxidized regenerated cellulose according to the manufacturer's instructions; and group C, the muscle defect was treated with Surgicel Snow absorbable oxidized regenerated cellulose according to the manufacturer's instructions. After application, 4-0 Prolene was used to mark the biopsy locations, and the wound was sutured with 5-0 nylon. The suture sites were disinfected with $10 \%$ povidine iodine and $70 \%$ alcohol, and a standard pellet diet and tap water were supplied during the experiment. After the operation, each rat's weight was measured and compared to that before the surgery, and the general condition of each was checked.

\section{Wound biopsy}

Four animals were randomly selected and sacrificed on the 3rd, 7th, and 27th days after surgery. The tissues were taken to include normal surrounding tissue with muscle defects marked with Prolene, fixed with 10\% formalin solution for more than 6 hours, and stained with hematoxylin and eosin stain.

\section{Histological evaluation}

A histological analysis was performed using an optical microscope, and each group was compared with the control group using seven parameters (foreign body giant cell, inflammatory cell [PMNL, macrophage] infiltration, granulation tissue, fibroblast proliferation and migration, neovascularization, extracellular matrix [ECM] formation [collagen deposition], and striated muscle repair [centronucleated cell]). The histological analysis was performed by a pathologist using a semi-quantitative scoring system that quantified each parameter ( 0 , absent; 1 , slight; 2 , moderate; 3 , extensive).

\section{Statistical analysis}

We analyzed the wound healing effect of each of the three hemostatic agents by screening for the parameters listed above. And the data on parameter using a semi-quantitative scoring system that quantified each parameter is expressed as mean \pm standard deviation. The Kruskal-Wallis test was conducted in each group. Statistical analysis was performed using SPSS version 23.0 (IBM Corp., Armonk, NY, USA) with $p$-values $<0.05$ considered statistically significant. 


\section{RESULTS}

\section{Statistical analysis}

The data using a semi-quantitative scoring system that quantified each parameter is expressed as mean \pm standard deviation (Tables 1-3).

\section{Histological evaluation}

Day 3

In the control group, fibroblast proliferation, inflammatory cell infiltration, ECM formation, and striated muscle repair were observed. These parameters were increased in groups A, B, and $\mathrm{C}$ compared to the control group. In particular, ECM formation (collagen deposition) and muscle regeneration were significantly increased in group A compared to groups B and C. The amount of granulation tissue was similar in all groups (Fig. 1).

\section{Day 7}

In the control group, fibroblast proliferation, inflammatory cell infiltration, ECM formation, and striated muscle repair were increased than day 3. Fibroblast proliferation and neovascularization were increased similarly in all groups compared to the

Table 1. On day 3, parameter of wound healing using a semi-quantitative scoring system

\begin{tabular}{lccccc}
\hline & Control group & Group A (Tachosil) & Group B (Surgicel Fibrillar) & Group C (Surgicel Snow) & $p$-value \\
\hline Foreign body giant cell & 0 & 0 & 0 & 0 & - \\
Inflammatory cell infiltration & $2.00 \pm 0.00$ & $2.33 \pm 0.58$ & $2.00 \pm 0.00$ & $2.33 \pm 0.58$ & 0.68 \\
Granulation tissue & $2.00 \pm 0.00$ & $2.00 \pm 0.00$ & $2.00 \pm 0.00$ & $1.00 \pm 0.00$ & - \\
Fibroblast proliferation & $1.00 \pm 0.00$ & $2.00 \pm 0.00$ & $1.00 \pm 0.00$ & $1.00 \pm 00$ & 0.029 \\
Neovascularization & $1.00 \pm 0.00$ & $1.00 \pm 0.00$ & $3.00 \pm 0.00$ & $3.00 \pm 0.00$ & 0.00 \\
ECM formation & $1.00 \pm 0.00$ & $3.00 \pm 0.00$ & $1.50 \pm 0.71$ & $1.67 \pm 0.58$ & 0.057 \\
Striated muscle repair & $0.50 \pm 0.71$ & $3.00 \pm 0.00$ & & & \\
\hline
\end{tabular}

Values are presented as mean \pm SD. Scoring: 0, absent; 1 , slight; 2, moderate; 3, extensive.

ECM, extracellular matrix.

$p$-values $<0.05$ considered statistically significant.

Table 2. On day 7, parameter of wound healing using a semi-quantitative scoring system

\begin{tabular}{lccccc}
\hline & Control group & Group A (Tachosil) & Group B (Surgicel Fibrillar) & Group C (Surgicel Snow) & $p$-value \\
\hline Foreign body giant cell & 0 & $0.67 \pm 0.58$ & $2.50 \pm 0.71$ & $3.00 \pm 0.00$ & 0.068 \\
Inflammatory cell infiltration & $3.00 \pm 0.00$ & $3.00 \pm 0.00$ & $3.00 \pm 0.00$ & $3.00 \pm 0.00$ & - \\
Granulation tissue & $3.00 \pm 0.00$ & $3.00 \pm 0.00$ & $3.00 \pm 0.00$ & $3.00 \pm 0.00$ & $3.00 \pm 0.00$ \\
Fibroblast proliferation & $2.00 \pm 0.00$ & $3.00 \pm 0.00$ & $3.00 \pm 0.00$ & $3.00 \pm 0.00$ & $3.00 \pm 0.00$ \\
Neovascularization & $2.00 \pm 0.00$ & $3.00 \pm 0.00$ & $1.00 \pm 0.00$ & $2.00 \pm 0.00$ & 0.046 \\
ECM formation & $2.00 \pm 0.00$ & $2.00 \pm 0.00$ & $2.50 \pm 0.71$ & $3.00 \pm 0.00$ & 0.046 \\
Striated muscle repair & $2.00 \pm 0.00$ & $2.00 \pm 0.00$ & & & 0.11 \\
\hline
\end{tabular}

Values are presented as mean \pm SD. Scoring: 0 , absent; 1 , slight; 2, moderate; 3 , extensive.

ECM, extracellular matrix.

$p$-values $<0.05$ considered statistically significant.

Table 3. On day 27, parameter of wound healing using a semi-quantitative scoring system

\begin{tabular}{lccccc}
\hline & Control group & Group A (Tachosil) & Group B (Surgicel Fibrillar) & Group C (Surgicel Snow) & $p$-value \\
\hline Foreign body giant cell & 0 & $1.00 \pm 0.00$ & $1.33 \pm 0.58$ & $1.67 \pm 0.58$ & 0.072 \\
Inflammatory cell infiltration & $0.50 \pm 0.71$ & $1.00 \pm 0.00$ & $1.33 \pm 0.58$ & $1.33 \pm 0.58$ & 0.31 \\
Granulation tissue & 0 & $0.33 \pm 0.58$ & $0.67 \pm 0.58$ & $1.00 \pm 0.00$ & 0.16 \\
Fibroblast proliferation & 0 & $0.33 \pm 0.58$ & $0.67 \pm 0.58$ & $1.00 \pm 0.00$ & 0.16 \\
Neovascularization & $1.00 \pm 0.00$ & $0.33 \pm 0.58$ & $0.67 \pm 0.58$ & $1.00 \pm 0.00$ & 0.27 \\
ECM formation & $1.00 \pm 0.00$ & $1.67 \pm 0.58$ & $2.00 \pm 0.00$ & $0.00 \pm 0.00$ & 0.074 \\
Striated muscle repair & $0.50 \pm 0.71$ & 0 & $0.67 \pm 0.58$ & $1.67 \pm 0.58$ & 0.076 \\
\hline
\end{tabular}

Values are presented as mean \pm SD. Scoring: 0, absent; 1, slight; 2, moderate; 3, extensive.

ECM, extracellular matrix.

$p$-values $<0.05$ considered statistically significant. 

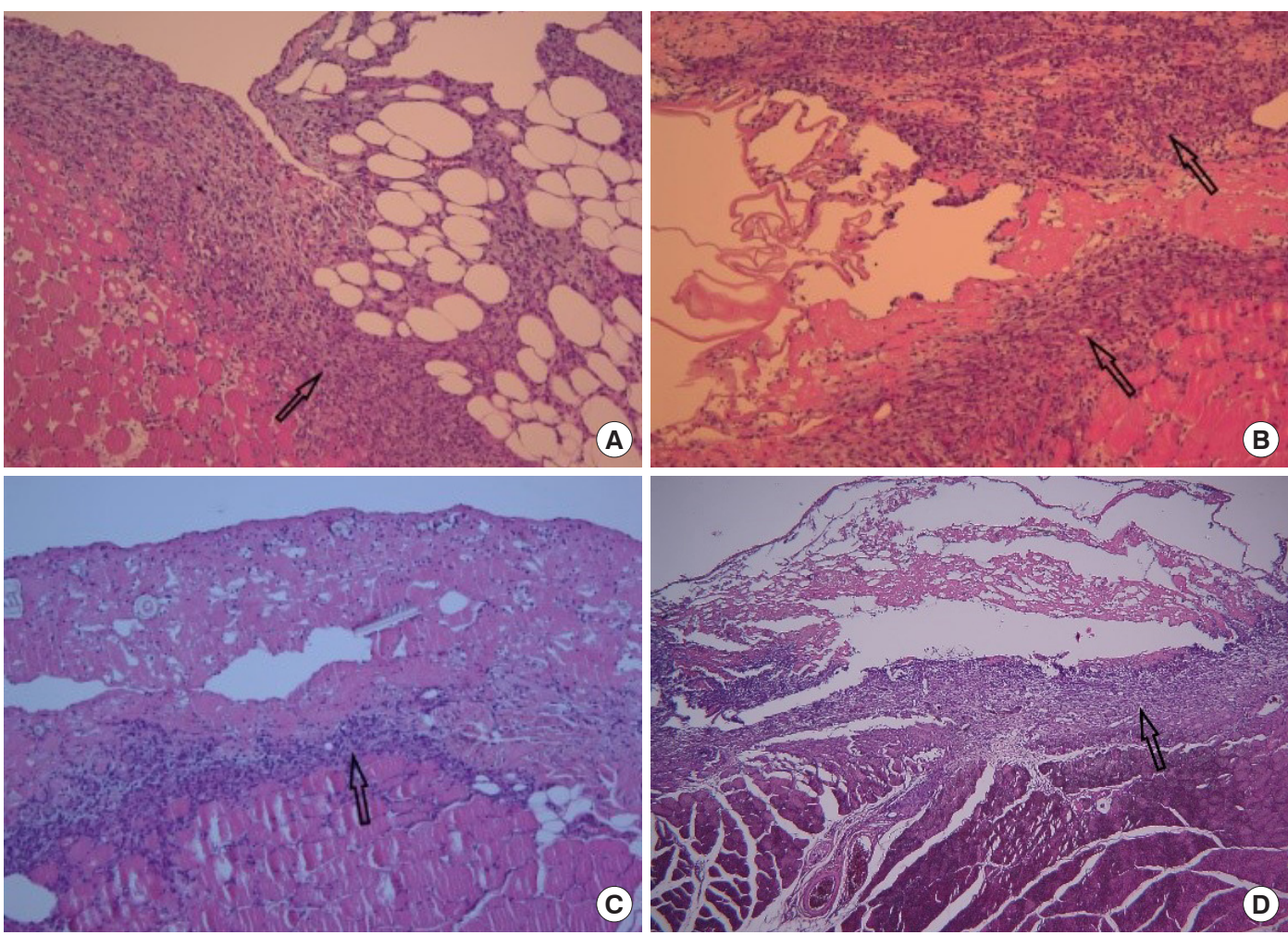

Fig. 1. Three days after wound induction $(\mathrm{H} \& \mathrm{E}, \times 100)$. (A) Control group, fibroblast proliferation, inflammatory cell infiltration, extracellular matrix (ECM) formation, and striated muscle repair (arrow) were observed. (B) Group A, ECM formation and muscle regeneration (arrows) were significantly increased compared to groups B and C. Groups B (C) and C (D), ECM formation and muscle regeneration (arrow) were less than in group A, but increased compared to the control group.

control group. Foreign body giant cell increased in the order of group A, B, and C. The amount of granulation tissue was similar among all groups (Fig. 2).

\section{Day 27}

In the control group, wound healing was completed and little inflammatory cell infiltration was observed. The group A was observed similarly to the control group. However, foreign body giant cell and inflammatory cell infiltration and the amount of granulation tissue increased continuously in the order of group $\mathrm{B}$ and $\mathrm{C}$. The muscle regeneration lasted until the last stage in group C. The final amount of ECM formed was similar among all groups (Fig. 3).

\section{DISCUSSION}

Wound healing occurs through inflammation, proliferation, and maturation phases. The hemostasis phase occurs within about 10-15 minutes after the wound occurs and is often included in the inflammatory phase. Upon the initial tissue damage, the damaged blood vessels contract, causing the platelet aggregation and coagulation cascade activation to produce a platelet plug. The platelet plug is a physiological barrier that prevents bleeding, bacterial infection, and water loss that serves as a provisional matrix. The cells in the platelet plug contain a variety of growth factors that induce the influx of inflammatory cells into the wound site and activate fibroblasts, vascular endothelial cells, and macrophages to begin the inflammatory phase. Platelets increase vascular permeability through serotonin. Neutrophils are the first white blood cells to enter the wound, and their values peak within 24 hours. They cause acute inflammation and play an immunological role in preventing bacterial contamination and infection. They also secret protease to remove damaged ECM and tissue. Next, monocytes enter, are activated and converted to macrophages, and act as the major cells that destroy foreign bodies or bacteria within 48-72 hours. They secrete several growth factors and offer phagocytosis, which induces ECM formation and the proliferation of fibroblasts, vascular smooth muscle cells, and vascular endothelial cells. Finally, lymphocytes appear after 72 hours. When the wound exits the inflammatory phase, the next proliferation phase begins. As macrophage numbers decrease, fibroblasts, vascular endothelial cells, and keratinocytes are produced and secrete growth factors. These growth factors stimulate ECM 

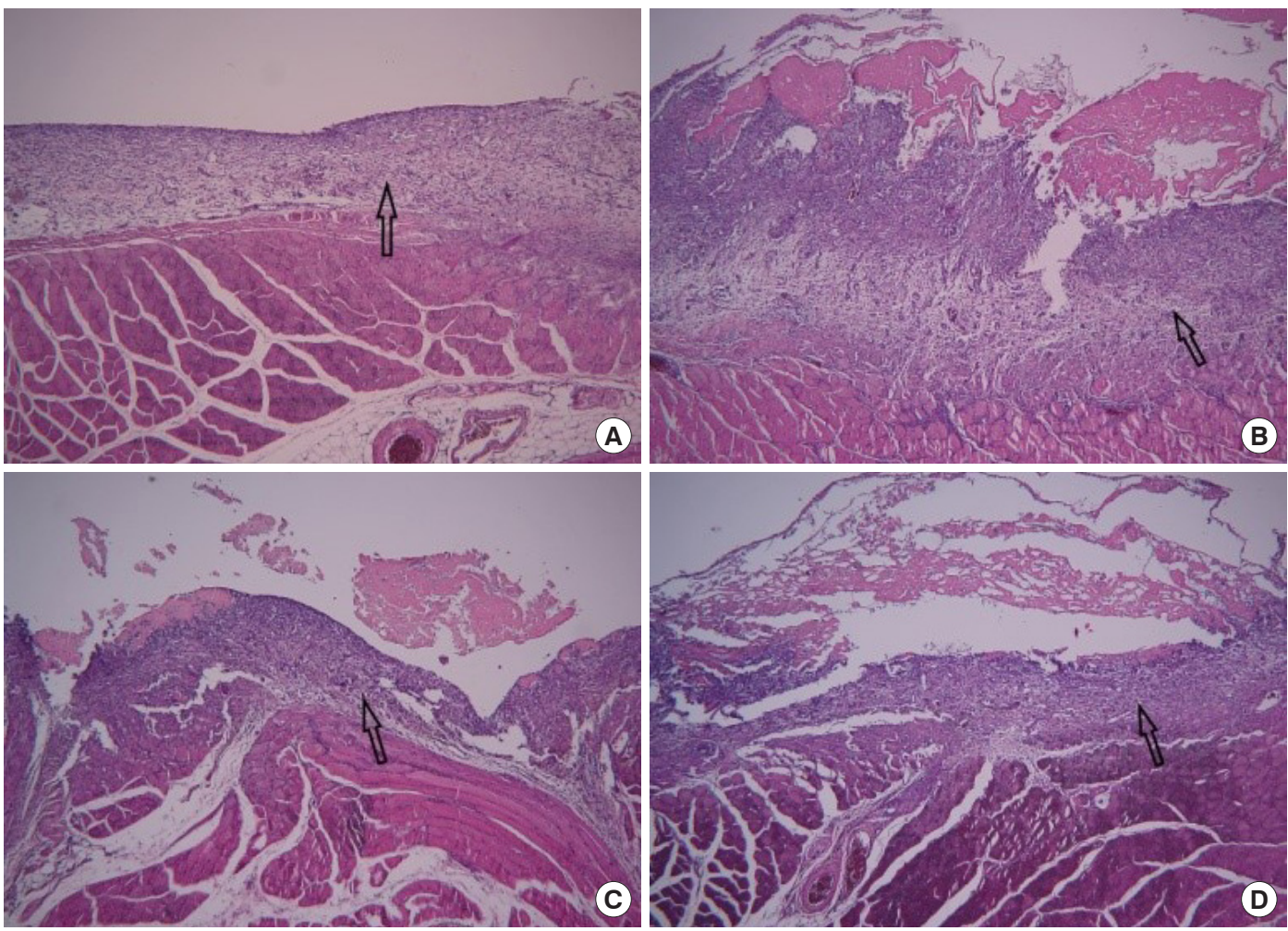

Fig. 2. Seven days after wound induction $(\mathrm{H} \& \mathrm{E}, \times 40)$. (A) Control group, fibroblast proliferation, inflammatory cell infiltration, extracellular matrix formation, and striated muscle repair were increased than day 3. Sufficient granulation tissue (arrow) is formed. (B) Group A, fibroblast proliferation, neovascularization and the amount of granulation tissue (arrow) were increased compared to the control group. Foreign body giant cells were rarely observed. Groups B (C) and C (D), fibroblast proliferation and neovascularization were increased similarly in all groups compared to the control group. Foreign body giant cell (arrow) increased in the order of groups A, B, and C.
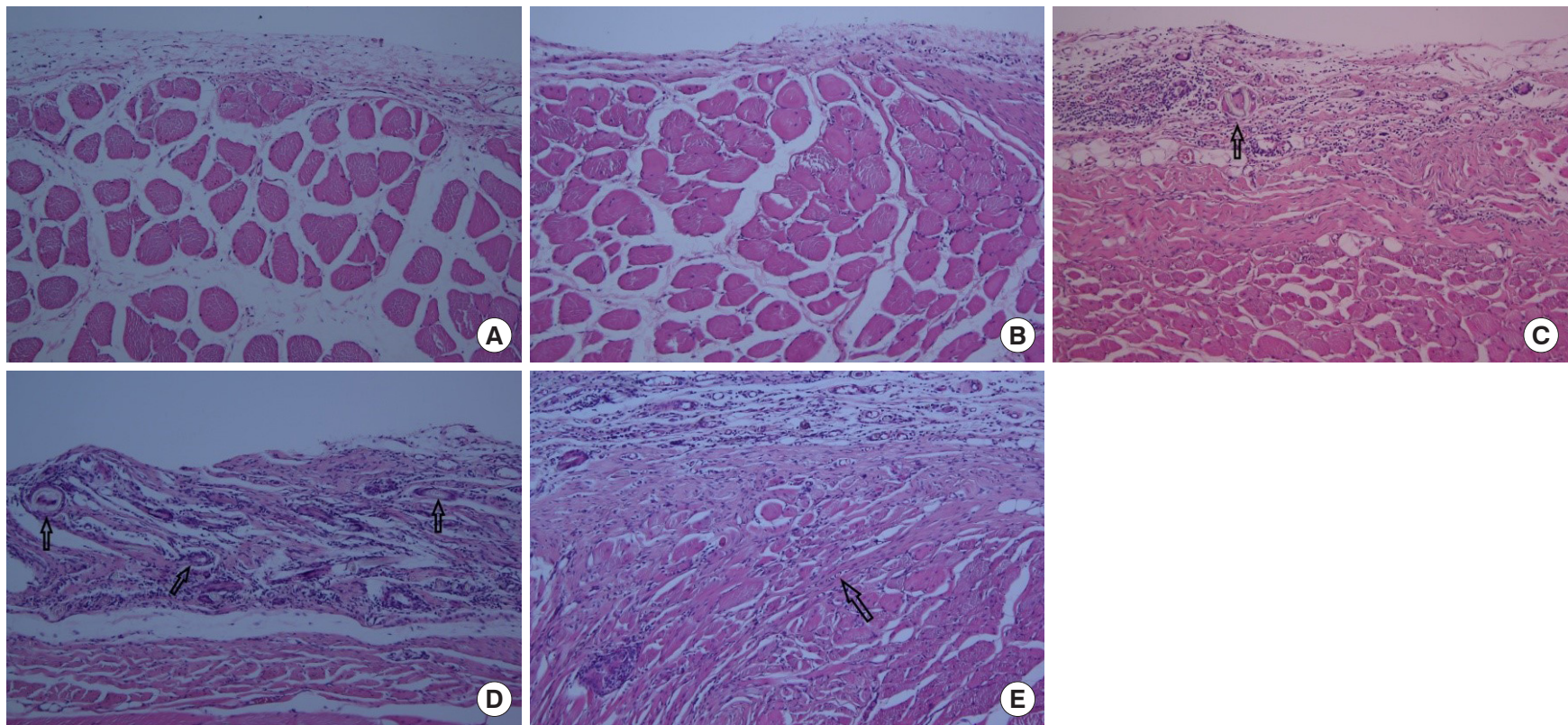

Fig. 3. Twenty-seven days after wound induction $(\mathrm{H} \& \mathrm{E}, \times 100)$. (A) Control group, Wound healing was completed and little inflammatory cell infiltration was observed. (B) Group A, it was observed similarly to the control group. (C) Group B, foreign body giant cell (arrow) and inflammatory cell infiltration and the amount of granulation tissue increased continuously in the order of groups B and C. (D) Group C, foreign body giant cell (arrows) and inflammatory cell infiltration were significantly increased. (E) Group C, the muscle regeneration (arrow) lasted until the last stage in group C. 
production and neovascularization. The proliferative phase consists of epithelialization, ECM deposition, vascularization, and wound contraction. In the maturation phase, mature scars are formed by collagen reconstruction through structural modifications [5-7]. If the inflammatory phase persists without timely and orderly progression, wound healing may become delayed or severe scarring may occur, and the wound can become chronic, presenting clinical problems and frequently causing morbidity and mortality $[6,8]$.

Wound healing is a dynamic process that is difficult to distinguish clearly and requires consistent measurements. Wound healing is usually assessed by clinical features and biochemical and histological parameters [6]. Histological evaluations should include the basic components of the healing process, including angiogenesis (neovascularization), inflammation (inflammatory cell infiltration), fibroplasia and restoration of the connective tissue matrix (collagen deposition), wound contraction and remodeling, epithelialization, and differentiation $[5,6,8]$. A quantitative scoring system is highly specific and standardized; however, in most cases, it is difficult to use because it is a difficult to objectify the exact interval between two values. Therefore, in biochemical research, semi-quantitative scoring systems are widely used to measure and express the degree of change between scales [6]. In this study, a comparative study using semiquantitative scoring system was also conducted.

Methods of hemostasis include: mechanical techniques (compression, suture, ligation, etc.); thermal techniques (electrocautery, laser, etc.); and chemical techniques (pharmacotherapyhypotensive anesthesia, epinephrine, vitamin K, desmopressin/ topical hemostatic-collagen, cellulose, gelatin, thrombin, fibrin sealant, etc.) [1]. Of these methods, topical hemostatic agents are used when other methods are impractical [2]. Ideally, topical hemostatic agents should be easy to use; quickly and effectively achieve hemostasis; and be nonantigenic, fully absorbable, and inexpensive. However, such hemostatic agents do not exist. Therefore, surgeons must be aware of the mechanisms, advantages, and disadvantages of various topical hemostatic agents to ensure their appropriate use [1,9]. Samudrala [1] classified topical hemostatic agents as follows according to their mechanism of action: active (collagen, cellulose, gelatin, etc.); passive (thrombin, etc.); or tissue sealant (fibrin sealant, polyethylene glycol, albumin/glue, etc.). Achneck et al. [9] classified topical hemostatic agents according to their components as: physical (bone wax, ostene, etc.); absorbable (gelatin, oxidized cellulose, microfibrillar collagen, etc.); biologic (thrombin, fibrin sealant, platelet sealant, etc.); and synthetic (polyethylene glycol hydrogels, cyanoacrylates, glutaraldehyde cross-linked albumin, etc.). The mechanism, advantages, and disadvantages of clinically frequently used hemostatic agents are summarized in Table $4[1,3,9]$. Topical hemostatic agents do not adhere strongly to wet tissues and have little effect on actively bleeding wounds. And, their dissolution does not indicate their disappearance from the site of application. Although hemostatic agents are dissolved in a short period of time, the absorption of their residue and wound healing involve another process. Surgeons believe that it will be absorbed immediately, but it may remain unabsorbed depending on environmental factors, so it is preferable to use the minimum amount needed. In addition, topical hemostatic agents may increase infection, so minimal amounts should be used in cases of a risk of contamination [2].

Surgicel Fibrillar used in this experiment is very loose and relatively thicker $(>5 \mathrm{~mm}$ ) than the original. Surgicel Snow is thick, tightly woven, and denser than Surgicel Fibrillar [10]. Surgicel consists of uronic acid components and fibrous residue. This fibrous residue is processed by macrophages and is not associated with foreign body giant cell, so it has low antigenicity. In addition, it has fibrin affinity with hemostatic potential and acts as a scaffold for fibrin deposition and clot formation [11]. Above all, it is widely used because of its low cost and easy application, and it is known to take $2-6$ weeks to be absorbed $[2,12]$. Surgicel inhibits bacterial growth by preventing the influx of sodium hydroxide and lowering the $\mathrm{pH}$ to about 2.5 [13]. Surgicel is effective against a broad spectrum of bacteria and antibiotic-resistant bacteria, and since bacteria have a

Table 4. The mechanism, advantages, and disadvantages of clinically frequently used hemostatic agents

\begin{tabular}{|c|c|c|c|}
\hline Hemostatic agent & Mechanism of action & Advantages and recommendations & Disadvantages and cautions \\
\hline Gelatin foams & $\begin{array}{l}\text { Swollen gelatin restricts blood flow and } \\
\text { provides physical matrix for clotting } \\
\text { initiation }\end{array}$ & $\begin{array}{l}\text { Absorbed within } 4-6 \text { weeks } \\
\text { Nonantigenic } \\
\text { Neutral pH allows use with biologic } \\
\text { hemostatic agents }\end{array}$ & $\begin{array}{l}\text { Can cause compressive complications when used } \\
\text { near nerves or in confined spaces (swelling up to } \\
2 \text { times the initial volume) } \\
\text { May embolize if in an intravascular compartment }\end{array}$ \\
\hline Regenerated oxidized cellulose & $\begin{array}{l}\text { Provide physical matrix for clotting } \\
\text { initiation }\end{array}$ & $\begin{array}{l}\text { Low pH has antimicrobial effect } \\
\text { Very good handling } \\
\text { Does not stick to instruments } \\
\text { Absorbed within 2-6 weeks }\end{array}$ & $\begin{array}{l}\text { Must not be used with other biologic hemostatic } \\
\text { agents because of low pH } \\
\text { Low pH may increase inflammation of surrounding } \\
\text { tissue } \\
\text { Can cause compressive complications when used } \\
\text { near nerves or in confined spaces }\end{array}$ \\
\hline
\end{tabular}


growth limit at $\mathrm{pH} 4.4-4.9$, it does not act on the mechanism itself like antibiotics do [10]. However, lowering the $\mathrm{pH}$ reportedly causes strong inflammatory reactions and delays wound healing $[2,12,14]$.

The Tachosil used in this experiment is an absorbable fibrin sealant patch that consists of a combination of fibrin sealant (tissue sealant) and collagen (passive hemostatic agent) [1,3]. Tachosil is absorbed within about 12 weeks and reportedly reduces scar formation and adhesions [3]. The fibrin sealant is composed of human fibrinogen, human thrombin, $\mathrm{Ca}^{2+}$, and factor XIII; dissolved fibrinogen and thrombin contact the fluid and act at the end of the coagulation cascade. Thrombin cleaves fibrinogen into fibrin monomers and activates factor XIII to factor XIIIa together with $\mathrm{Ca}^{2+}$. Fibrin monomers form unstable and soluble fibrin polymers, which are then cross-linked by factor XIIIa to become stable and insoluble fibrin polymers, resulting in fibrin clots $[3,9,15]$. Fibrin regulates the inflammatory phase by regulating monocyte and macrophage activity as well as hemostasis in the wound healing process. It also acts as a growth factor, directly affecting angiogenesis and promoting fibroblast formation. Through this action, it has a good effect on wound healing [7]. Tachosil has the advantages of hemostasis, providing tissue sealing, supporting sutures, preventing adhesions, protecting nerves, slowly releasing medications, and promoting wound healing, and is clinically used for hemostasis, adherence purposes during skin grafting, nerve anastomosis, bone sealing, dura sealing, and mastectomy to reduce seroma formation, slowly releasing medications, including antibiotics, growth factors, and chemotherapeutic agents [3,15-17]. However, if a contaminated or infected area or active infections are present, its use should be avoided. There are also problems with immunologic reactions and the transmission of infectious disease (human immunodeficiency virus, hepatitis, parvovirus B19, and Creutzfeldt-Jakob disease) $[3,15,17]$. As a solution, viral inactivation using nanofiltration, heat treatment, solvent detergent cleansing, autologous donation techniques, and recombination production methods are used [17].

In this study, although the data values were not statistically significant, differences in each group were observed on histological evaluation. The Surgicel and Tachosil groups showed an increase in all parameters compared to the control group. Each increased inflammation as a foreign body but did not significantly interfere with the wound healing process. The increase in foreign body giant cell and inflammatory cell infiltration in the Surgicel group is thought to be due to low $\mathrm{pH}$; in contrast, Tachosil induces a less serious foreign body reaction. In the Tachosil group, the initial ECM formation amount was higher than that of other groups, but it is difficult to distinguish the newly generated amount accurately because it is mixed with collagen in the product components. As the product components were absorbed later, the amount of ECM formation was similar in all groups. However, due to the fibrin action seen in the Tachosil group, the wound healing proceeded faster than in the other groups. The authors believe that this study was not statistically significant at below $5 \%$. But, considering that it is a significant value below $10 \%$, it is expected that statistically significant results will be obtained if the number of samples increases.

In conclusion, we recommend the use of Tachosil because it features a less severe foreign body reaction than Surgicel and facilitates faster wound healing due to the fibrin action.

\section{NOTES}

\section{Conflict of interest}

No potential conflict of interest relevant to this article was reported.

\section{Ethical approval}

The study was approved by the Institutional Animal Care and Use Committee of Wonkwang University (IACUC No. WKU 19-53).

\section{ORCID}

Hyun Sik Yoon https://orcid.org/0000-0002-2294-5465

Young Cheon Na https://orcid.org/0000-0003-3136-0351

Keum Ha Choi https://orcid.org/0000-0001-8617-4869

Woo Hoe Huh https://orcid.org/0000-0002-1379-7122

Ji Min Kim https://orcid.org/0000-0002-9819-0775

\section{REFERENCES}

1. Samudrala S. Topical hemostatic agents in surgery: a surgeon's perspective. AORN J 2008;88:S2-11.

2. Tomizawa Y. Clinical benefits and risk analysis of topical hemostats: a review. J Artif Organs 2005;8:137-42.

3. Rickenbacher A, Breitenstein S, Lesurtel M, Frilling A. Efficacy of TachoSil a fibrin-based haemostat in different fields of surgery: a systematic review. Expert Opin Biol Ther 2009;9:897907.

4. Vidinsky B, Gal P, Toporcer T, Longauer F, Lenhardt L, Bobrov $\mathrm{N}$, et al. Histological study of the first seven days of skin wound healing in rats. Acta Vet Brno 2006;75:197-202.

5. Amin ZA, Ali HM, Alshawsh MA, Darvish PH, Abdulla MA. Application of Antrodia camphorata promotes rat's wound healing in vivo and facilitates fibroblast cell proliferation in vi- 
tro. Evid Based Complement Alternat Med 2015;2015:317693.

6. Gupta A, Kumar P. Assessment of the histological state of the healing wound. Plast Aesthet Res 2015;2:239-42.

7. Laurens N, Koolwijk P, de Maat MP. Fibrin structure and wound healing. J Thromb Haemost 2006;4:932-9.

8. Lazarus GS, Cooper DM, Knighton DR, Margolis DJ, Pecoraro RE, Rodeheaver G, et al. Definitions and guidelines for assessment of wounds and evaluation of healing. Arch Dermatol 1994;130:489-93.

9. Achneck HE, Sileshi B, Jamiolkowski RM, Albala DM, Shapiro ML, Lawson JH. A comprehensive review of topical hemostatic agents: efficacy and recommendations for use. Ann Surg 2010; 251:217-28.

10. Spangler D, Rothenburger S, Nguyen K, Jampani H, Weiss S, Bhende S. In vitro antimicrobial activity of oxidized regenerated cellulose against antibiotic-resistant microorganisms. Surg Infect (Larchmt) 2003;4:255-62.

11. Pierce A, Wilson D, Wiebkin O. Surgicel: macrophage process- ing of the fibrous component. Int J Oral Maxillofac Surg 1987; 16:338-45.

12. Soares LP, Oliveira MG, Pinheiro AL, Fronza BR, Maciel ME. Effects of laser therapy on experimental wound healing using oxidized regenerated cellulose hemostat. Photomed Laser Surg 2008;26:10-3.

13. Dineen P. The effect of oxidized regenerated cellulose on experimental infected splenotomies. J Surg Res 1977;23:114-25.

14. Olson RA, Roberts DL, Osbon DB. A comparative study of polylactic acid, Gelfoam, and Surgicel in healing extraction sites. Oral Surg Oral Med Oral Pathol 1982;53:441-9.

15. Spotnitz WD. Commercial fibrin sealants in surgical care. Am J Surg 2001;182(2 Suppl):8S-14S.

16. Matras $\mathrm{H}$. The use of fibrin sealant in oral and maxillofacial surgery. J Oral Maxillofac Surg 1982;40:617-22.

17. Spotnitz WD, Falstrom JK, Rodeheaver GT. The role of sutures and fibrin sealant in wound healing. Surg Clin North Am 1997; 77:651-69. 